\title{
Absorbers: Definitions, Properties and Applications
}

\author{
G. BELITSKII ${ }^{\dagger}$ \\ Ben-Gurion University of the Negev, P.O. Box 653, Beer-Sheva 84105, Israel
}

(Received 12 January 1997; Revised 26 March 1997)

\begin{abstract}
Roughly speaking, the absorber is a set, which includes, after finite number of initial states, each trajectory of a transformation of space into itself. This paper deals with the exact definition of absorbers for linear operators, the study of the properties, the applications to "classical" dynamics and to solvability of operator equations. It is expected that the description of the structure of absorbers will add new insights to the recent discussion of nature and content of notion of attractiveness for nonlinear dynamics.
\end{abstract}

Keywords: Absorbers, Induced dynamics, Functional equations

\section{INTRODUCTION}

We consider linear operators in the spaces of continuous or smooth functions on topological spaces or, correspondingly, on smooth manifolds.

More exactly, let $X$ and $Y$ be topological spaces, and $\mathcal{E}_{1}, \mathcal{E}_{2}$ be linear topological spaces. Denote by $\xi_{0}$ and $\eta_{0}$ the sheaves of germs of continuous mappings $X \rightarrow \mathcal{E}_{1}$ and $Y \rightarrow \mathcal{E}_{2}$ correspondingly. It means (see [6]) that the space $\Gamma\left(\xi_{0} \mid U\right)$ of local sections on $U \subset X$ is the set of all continuous mappings $U \rightarrow \mathcal{E}_{1}$. Similarly, $\psi \in \Gamma\left(\eta_{0} \mid V\right)$, $V \subset Y$ means that $\psi$ is a continuous mapping $V \rightarrow \mathcal{E}_{2}$.

Further, we consider the subsheaves $\xi \subset \xi_{0}$ and $\eta \subset \eta_{0}$. It means that $\Gamma(\xi \mid U), U \subset X$, and $\Gamma(\eta \mid V) \subset$ $\Gamma\left(\eta_{0} \mid V\right), V \subset Y$, are corresponding subspaces.

We do not assume the spaces $\Gamma(\xi)$ and $\Gamma(\eta)$ of global sections to be endowed with any topology.

\footnotetext{
${ }^{\dagger}$ Research is supported by Guastello Foundation.
}

Consider some linear mapping $T: \Gamma(\xi) \rightarrow \Gamma(\eta)$. We show that $T$ induces some "dynamics" on the set of all subsets $S \subset X$. In terms of this dynamics, we single out the definition of absorbers. The main property of these subsets is that they contain all obstacles to the solvability of the equation

$$
T \varphi=\gamma,
$$

with given $\gamma \in \Gamma(\eta)$ and unknown $\varphi \in \Gamma(\xi)$. Connections between absorbers and "classical" dynamics of mappings are also discussed.

\section{DEFINITIONS}

\subsection{Induced Dynamics}

For a section $\varphi \in \Gamma(\xi)$ we set

$$
N(\varphi)=\{y \in Y \mid(T \varphi)(y)=0\} .
$$


Obviously, $N(\varphi) \subset Y$ is a closed subset. For a subset $S \subset Y$ set

$$
T_{*}(S)=\bigcap N(\varphi), \quad \varphi \mid S=0 .
$$

In other words, $y \in T_{*}(S)$ if and only if $(T \varphi)(y)=0$ for all sections $\varphi \in \Gamma(\xi)$ vanishing on $S$.

It may happen that $T_{*}(S)=\emptyset$ for a subset $S \subset Y$. Obviously,

$$
T_{*}(X)=Y, \quad(\mathrm{id})_{*}(S)=S .
$$

Here id $: \Gamma(\xi) \rightarrow \Gamma(\xi)$ is identity mapping.

\section{Example 2.1 Let}

$$
\Gamma(\xi)=C(X), \quad \Gamma(\eta)=C(Y) .
$$

It means that $\xi=\xi_{0}, \eta=\eta_{0}$ with $\mathcal{E}_{1}=\mathcal{E}_{2}=\mathbb{R}$ (see Introduction). Given continuous $f: Y \rightarrow X$ let us set

$$
(T \varphi)(y)=\varphi(f(y)), \quad \varphi \in C(X) .
$$

Then

$$
T_{*}(S) \supset f^{-1}(S) .
$$

Example 2.2 More generally, let $f_{t}: Y \rightarrow X$, $t \in I \subset \mathbb{R}$ be a family of continuous mappings. Assume that measure $\mu$ on $I$ and continuous mapping $g: Y \times \mathbb{R} \rightarrow \mathbb{R}$ are such that

$$
(T \varphi)(y)=\int g(t, y) \cdot \varphi\left(f_{t} y\right) \mathrm{d} \mu \in C(Y)
$$

for all $C(X)$. In other words, $T$ acts from $C(X)$ to $C(Y)$. Then

$$
T_{*}(S) \supset \bigcap_{t \in I} f_{t}^{-1}(S) .
$$

\subsection{Some Properties of the Induced Operation}

Proposition 2.3 (a) The subset $T_{*}(S) \subset Y$ is closed for any $S \subset X$;

(b) $S_{1} \subset S_{2} \Rightarrow T_{*}\left(S_{1}\right) \subset T_{*}\left(S_{2}\right)$;

(c) $T_{*}\left(S_{1} \cup S_{2}\right) \supset T_{*}\left(S_{1}\right) \cup T_{*}\left(S_{2}\right)$;

(d) $T_{*}\left(S_{1} \cap S_{2}\right) \subset T_{*}\left(S_{1}\right) \cap T_{*}\left(S_{2}\right)$.
These properties are obvious.

Consider three sheaves $\xi, \eta, \zeta$ on $X, Y$ and $Z$ correspondingly. Let $T: \Gamma(\xi) \rightarrow \Gamma(\eta)$ and $H: \Gamma(\eta) \rightarrow$ $\Gamma(\zeta)$ be some mappings.

Proposition 2.4 The inclusion

$$
(T H)_{*}(S) \supset T_{*} H_{*}(S), \quad S \subset X,
$$

holds.

Indeed,

$$
\varphi_{1}\left|S=\varphi_{2}\right| S \Rightarrow H \varphi_{1}\left|H_{*}(S)=H \varphi_{2}\right| H_{*}(S) .
$$

Hence

$$
T H \varphi_{1}\left|T_{*} H_{*}(S)=T H \varphi_{2}\right| T_{*} H_{*}(S) .
$$

It follows from this that

$$
T_{*} H_{*}(S) \subset(T H)_{*}(S) .
$$

In particular, in $\xi=\eta$, then one can consider powers of the mappings $T$ and $T_{*}$. It follows from Proposition 2.4 that

$$
\left(T^{n}\right)_{*}(S) \supset\left(T_{*}\right)^{n}(S), \quad n=0,1,2, \ldots
$$

\subsection{Absorbers}

Definition 2.5 A pair $(A, L)$ where $A \subset Y$ is a closed subset and $L: \Gamma(\eta) \rightarrow \Gamma(\xi)$ is a linear mapping is called an absorber for the mapping $T$ if the following properties hold:

(a) Invariance:

$$
(T L)_{*}(A) \supset A
$$

(b) Absorption: for any $y \in Y$ there exist a neighborhood $W \ni y$ and a number $n_{0}=n_{0}(y)$ such that

$$
W \subset\left((\mathrm{id}-T L)^{n}\right)_{*} A, \quad n \geq n_{0} .
$$

Example 2.6 Let $\Gamma(\xi)=\Gamma(\eta)=C(X)$ and

$$
f: X \rightarrow X, \quad g: X \rightarrow \mathbb{R}
$$


are continuous mappings. Then the mapping

$$
(T \varphi)(x)=g(x) \cdot \varphi(f(x))
$$

acts in $C(X)$. Let us fix some open covering

$$
X=\bigcup_{\alpha} U_{\alpha}
$$

and a numerical function $q(\alpha) \in \mathbb{R}$. Set

$$
A=A\left(\{q(\alpha)\},\left\{U_{\alpha}\right\}\right)=\bigcup_{\alpha} \bigcup_{n \geq q(\alpha)} f^{n}\left(U_{\alpha}\right) .
$$

Then the pair ( $A$,id) be an absorber for $T$.

Example 2.7 Let

$$
(T \varphi)(y)=\sum_{i=1}^{l} a_{i}(y) \varphi\left(f_{i} y\right), \quad a_{i} \in C(Y),
$$

where $f_{i}: Y \rightarrow X$ are continuous. Assume that $a_{1}(y) \neq 0(y \in Y)$ and that $f_{1}$ is a homeomorphism. Set

$$
\begin{aligned}
& A=\overline{\bigcup_{\alpha} \bigcup_{n \geq q(\alpha)}\left(f_{1}^{-1} f_{i_{1}} \cdots\left(f_{1}^{-1} f_{i_{n}}\right)\left(U_{\alpha}\right)\right.}, \quad i_{\nu} \in \overline{1, l}, \\
& (L \psi)(x)=\frac{1}{a_{1}\left(f_{1}^{-1} x\right)} \psi\left(f_{1}^{-1} x\right), \quad \psi \in C(Y) .
\end{aligned}
$$

Then $(A, L)$ is an absorber for $T$.

Consider the set $\{(A, L)\}$ of all absorbers for $T$ and denote

$$
\mathcal{A}(T)=\bigcap A
$$

\section{EXTENSION OF SOLUTIONS FOR THE OPERATOR EQUATIONS}

The section $\varphi_{0} \in \Gamma(\xi)$ is said to be a solution of $E q$. (1.1) on a subset $V \in Y$ if the difference $\gamma-T \varphi_{0}$ vanishes on $V$.

THEOREM 3.1 Let $(A, L)$ be an absorber. Then for any solution $\varphi_{0}$ of Eq. (1.1) on A there exists a solution (on $X$ ) which coincides with $\varphi_{0}$ on the subset $L_{*} A$.
Proof Consider the equation

$$
T L \psi=\tilde{\gamma}, \quad \tilde{\gamma}=\gamma_{0}-T \varphi_{0} .
$$

It is sufficient to prove that this equation has a solution $\psi \in \Gamma(\eta)$, vanishing on $A$. Then the section $\varphi=\varphi_{0}+L \psi$ is a solution we need.

To this end let us set

$$
\psi_{n}=\tilde{\gamma}_{+}+\widetilde{T} \psi_{n-1}, \quad n=1,2, \ldots, \quad \psi_{0}=0,
$$

with $\widetilde{T} \psi=\psi-T L \psi$.

First of all note that

$$
\psi_{n} \mid A=0, \quad n=0,1, \ldots .
$$

Indeed, $\psi_{1}=\gamma-T \varphi_{0}$. Since $\varphi_{0}$ is a solution on $A$, we have $\left(\gamma-T \varphi_{0}\right) \mid A=0$. Hence, $\psi_{1} \mid A=0$. Now we obtain (3.2) by induction. Namely, assume that $\psi_{n-1} \mid A=0$. Then $T L \psi_{n-1}$ vanishes on the subset $(T L)_{*} A \supset A$. Therefore, $\psi_{n}=\tilde{\gamma}+\psi_{n-1}-T L \psi_{n-1}$ coincides with $\psi_{n-1}$ on $A$, i.e. $\psi_{n} \mid A=0$.

Further, let $y_{0} \in Y$. Choose a neighborhood $W \ni y_{0}$ and a number $n_{0}(y)$ such that

$$
W \subset\left(\widetilde{T}^{n}\right)_{*} A, \quad n \geq n_{0} .
$$

Consider a local section $h \in \Gamma(\eta \mid W)$, setting

$$
h=\psi_{n} \mid W, \quad n \geq n_{0} .
$$

It is well defined. Indeed, let $n \geq n_{0}$. Then

$$
\psi_{n}=\sum_{k=0}^{n_{0}-1} \widetilde{T}^{k} \gamma+\widetilde{T}^{n_{0}} \psi_{n-n_{0}} .
$$

Since

$$
\psi_{n-n_{0}} \mid A=0,
$$

the section $\psi_{n}$ coincides with $\sum_{k=0}^{n_{0}-1} \widetilde{T}^{k} \gamma$ on $\left(\widetilde{T}^{n_{0}}\right)_{*} A \supset W$. In other words, $\psi_{n}$ is stabilized on $W$ under $n \geq n_{0}$.

Continuing this construction, we will build some open covering $Y=\bigcup_{\alpha} W_{\alpha}$ and local sections $h_{\alpha} \in \Gamma\left(\eta \mid W_{\alpha}\right)$. Obviously, these sections coincide 
on the intersection $W_{\alpha} \cap W_{\beta}$. Since $\eta$ is a sheaf, there exists a global section $\psi$ such that $\psi \mid W_{\alpha}=h_{\alpha}$. The section $\psi$ satisfies (3.1) and $\psi \mid A=0$.

In particular, assume that $\gamma \mid A=0$. Then the zero section $\varphi_{0}=0$ is a solution on $A$. Hence, we obtain

COROLlaRy 3.2 Let $(A, L)$ be an absorber. If the section $\gamma$ vanishing on $A$, then Eq. (1.1) has a solution $\varphi$ vanishing on $L_{*} A$.

Further, let $\left(A_{i}, L_{i}\right), i \in \overline{1, q}$ be some finite number of absorbers. Assume that $\varphi_{0}$ is a solution on the intersection $\bigcap_{i} A_{i}$ in some more strong sense. Namely,

$$
\gamma-T \varphi_{0}=\gamma_{1}+\cdots+\gamma_{q},
$$

where $\gamma_{i} \mid A_{i}=0, i \in \overline{1, q}$. Then we have

COROLlary 3.3 There exists a solution of (1.1) coinciding with $\varphi_{0}$ on the intersection $\bigcap_{i=1}^{q}\left(L_{i}\right)_{*} A_{i}$.

Proof Consider the following equations:

$$
T \varphi_{i}=\gamma_{i}, \quad i \in \overline{1, q} .
$$

Since $\gamma_{i} \mid A_{i}=0$, there exists a solution $\varphi_{i}$ vanishing on $\left(L_{i}\right)_{*} A_{i}$. Then the section $\varphi=\varphi_{0}+\varphi_{1}+\cdots+\varphi_{q}$ is a solution of (1.1) coinciding with $\varphi_{0}$ on the intersection $\bigcap_{i}\left(L_{i}\right)_{*} A_{i}$.

In the case that any section $\delta \in \Gamma(\eta)$ vanishing on $\bigcap_{i=1}^{q} A_{i}$ may be decomposed to the sum $\sum_{i=1}^{q} \gamma_{i}$, $\gamma_{i} \mid A_{i}=0$, the solvability on $\bigcap_{i} A_{i}$ implies a global one. In particular, if $\bigcap_{i=1}^{q} A_{i}=\emptyset$, then we obtain an existence theorem: for any $\gamma \in \Gamma(\eta)$ Eq. (1.1) has a solution $\varphi \in \Gamma(\xi)$.

\section{APPLICATIONS}

\subsection{Localization of Linear Functionals}

Let $T: \Gamma(\xi) \rightarrow \Gamma(\eta)$ be a linear mapping. Then one can define a conjugate mapping

$$
T^{*}:(\Gamma(\eta))^{0} \rightarrow(\Gamma(\xi))^{0}
$$

between dual spaces of all linear functionals:

$$
\left(T^{*} \varphi\right)(\mu)=\mu(T \varphi), \quad \mu \in(\Gamma(\eta))^{0} .
$$

Obviously,

$$
\gamma \in \operatorname{Im} T \Rightarrow \mu(\gamma)=0 \quad\left(\mu \in \operatorname{Ker} T^{*}\right) .
$$

In other words, $\operatorname{Im} T \subset\left(\operatorname{Ker} T^{*}\right)_{\perp}$.

We will say that a point $y_{0} \in Y$ belongs to the support of a functional $\mu \in(\Gamma(\eta))^{0}$ if for any neighborhood $U \ni y_{0}$ there exists a section $\gamma \in \Gamma(\eta)$ vanishing outside of $U$ such that

$$
\mu(\gamma) \neq 0 .
$$

The set supp $\mu \subset Y$ of all such points $y_{0}$ is a closed subset. It may happen that $\operatorname{supp} \mu=\emptyset$. However, in any case we have

\section{THEOREM 4.1 The inclusion}

$$
\operatorname{supp} \mu \subset \mathcal{A}(T) \quad\left(\mu \in \operatorname{Ker} T^{*}\right)
$$

holds.

Proof Let $y_{0} \notin A$. Since $A$ is closed, there exists a neighborhood $U \ni y_{0}$ such that $U \cap A=\emptyset$. Assume that $y_{0} \in \operatorname{supp} \mu, \mu \in \operatorname{Ker} T^{*}$. Consider a section $\gamma \in \Gamma(\eta)$ vanishing outside of $U$ and such that $\mu(\gamma) \neq 0$. Since $\gamma \mid A=0, \gamma \in \operatorname{Im} T$ (see Corollary 3.2). The latter contradicts with unequality $\mu(\gamma) \neq 0$.

In the most interesting cases both of the spaces $\Gamma(\xi)$ and $\Gamma(\eta)$ are endowed by some topology. Then one can consider subspaces $(\Gamma(\xi))^{*} C(\Gamma(\xi))^{0}$ and $(\Gamma(\eta))^{*} \subset(\Gamma(\eta))^{0}$ of continuous linear functionals. If the linear operator $T: \Gamma(\xi) \rightarrow \Gamma(\eta)$ is a continuous one, then the conjugated $T^{*}$ is so.

For instance, let $X$ and $Y$ be a locally compact topological spaces or smooth manifolds and let $\Gamma(\xi)=C^{k}(X), \Gamma(\eta)=C^{p}(Y)$ with some $p, k \in[0, \infty]$. They are endowed by a natural topology with the convergence of all derivatives up to $p$ (or $k$ ) on compact subsets. Then $(\Gamma(\xi))^{*}$ and $(\Gamma(\eta))^{*}$ are spaces of corresponding distributions with compact supports. In particular,

$$
\mu \in(\Gamma(\xi))^{*}, \quad \operatorname{supp} \mu=\emptyset \Rightarrow \mu=0 .
$$


It is well known (see [7]) that

$$
\overline{\operatorname{Im} T}=\left(\operatorname{Ker} T^{*}\right)_{\perp} .
$$

Assume that $\gamma \mid \mathcal{A}(T)=0$. It follows from Theorem 4.1 that $\gamma \in\left(\operatorname{Ker} T^{*}\right)_{\perp}$. Hence, we obtain

COROLlaRY 4.2 If a function $\gamma \in(\Gamma(\eta))^{*}$ vanishes on $\mathcal{A}(T)$ then $\gamma \in \overline{\operatorname{Im} T}$. In particular, if $\mathcal{A}(T)=\emptyset$, then $\operatorname{Im} T$ is dense in the space $\Gamma(\eta)$.

On the other hand, as considerations after Corollary 3.3 show, in "regular cases" section $\gamma$, vanishing in a neighborhood of the intersection of a finite number of absorbers belongs to $\operatorname{Im} T$.

Example 4.3 Let $X=\mathbb{R}^{1}$ and

$$
(T \varphi)(x)=\sum_{i=1}^{l} a_{i}(x) \varphi\left(\alpha_{i} x+\beta_{i}\right), \quad \varphi \in C\left(\mathbb{R}^{1}\right),
$$

with $\alpha_{i}>0$ and continuous coefficients $a_{i}(x) \neq 0$ $\left(x \in \mathbb{R}^{1}\right)$. Let

$$
\alpha_{1}=\cdots=\alpha_{q}=\alpha>\alpha_{i}, \quad i \in \overline{1, q},
$$

and

$$
\beta_{1}<\cdots<\beta_{q},
$$

with some $q \in \overline{1, l}$. Then $T$ has two families of absorbers $\left(A_{ \pm}(c), L_{ \pm}\right)$. Namely,

$$
A_{-}(c)=[c,+\infty), \quad c<c_{-} \equiv \min _{i>q} \frac{\beta_{i}-\beta_{1}}{\alpha-\alpha_{i}},
$$

with

$$
\left(L_{-} \psi\right)(x)=\frac{1}{a_{1}\left(\alpha^{-1}\left(x-\beta_{1}\right)\right)} \psi\left(\alpha^{-1}\left(x-\beta_{1}\right)\right)
$$

and

$$
A_{+}(c)=(-\infty, c], \quad c>c_{+} \equiv \max _{i>q} \frac{\beta_{i}-\beta_{q}}{\alpha-\alpha_{i}},
$$

with

$$
\left(L_{+} \psi\right)(x)=\frac{1}{a_{q}\left(\alpha^{-1}\left(x-\beta_{q}\right)\right)} \psi\left(\alpha^{-1}\left(x-\beta_{q}\right)\right) .
$$

Hence,

$$
\mathcal{A}(T) \subset\left[c_{-}, c_{+}\right] .
$$

In the case $c_{+}<c_{-}$it means that $\mathcal{A}(T)=\emptyset$. Thus, any function $\gamma$ vanishing on the interval $\left[c_{-}, c_{+}\right]$, belongs to the closer of $\operatorname{Im} T$. In other words,

$$
\mu \in \operatorname{Ker} T^{*} \Rightarrow \operatorname{supp} \mu \subset\left[c_{-}, c_{+}\right] .
$$

Moreover, if Eq. (1.1) is solvable on an inverval $[a, b]$ with $a<c_{-}, b>c_{+}$, then $\gamma \in \operatorname{Im} T$. In particular, in the case $c_{-}>c_{+}$the operator $T: C(\mathbb{R}) \rightarrow C(\mathbb{R})$ is surjective (see [2]).

\subsection{Absorbers and Dynamics of Mappings}

Let $f: X \rightarrow X$ be a continuous mapping. Consider the linear operator

$$
H_{f}(\varphi)(x)=\varphi(x)-\varphi(f(x)), \quad \varphi \in C(X) .
$$

There is a connection between absorbers for $H_{f}$ and dynamical behavior of the mapping $f$ : any absorber contains all periodic points $\operatorname{Per}(f)$ and, moreover, all "attracting" points. Namely, let us remind that a point $x_{0} \in X$ is called $\omega$-limited for $f$ if there is some $z \in X$ and a sequence $\left\{n_{k}\right\} \subset \mathbb{Z}_{+}$ such that

$$
f^{n_{k}} z \rightarrow x_{0}, \quad k \rightarrow \infty
$$

If $f$ is a homeomorphism, then $\alpha$-limited point is a $\omega$-limited one for $f^{-1}$. Denote by $\Omega_{+}(f)$ the set of all $\omega$-limited points. In the case $f$ is a homeomorphism denote by $\Omega_{-}(f)$ the set of all $\alpha$-limited points.

THEOREM 4.4 Let $X$ be a normal topological space. Then the inclusion

$$
\Omega_{+}(f) \subset \mathcal{A}\left(H_{f}\right)
$$

holds.

Proof Let $x_{0} \in \Omega_{+}(f)$ and assume that $x_{0} \notin A$ for some absorber $(A, L)$. Then there exists a 
neighborhood $U \ni x_{0}$ such that $\bar{U} \cap A=\emptyset$. Let $\gamma \in C(X)$ be a nonnegative function such that

$$
\gamma|\bar{U}=1, \quad \gamma| A=0 .
$$

Due to Corollary 3.2, $\gamma \in \operatorname{Im} T$. Let $\varphi \in C(X)$ be a solution, i.e.

$$
\varphi(x)-\varphi(f(x))=\gamma(x) \quad(x \in X) .
$$

Then

$$
\begin{aligned}
& \varphi(x)=\varphi\left(f^{n}(x)\right)+\sum_{j=0}^{n-1} \gamma\left(f^{j}(x)\right) ; \quad x \in X, \\
& n=1,2, \ldots
\end{aligned}
$$

Now let $f^{n_{k}} z \rightarrow x_{0}$. Then we obtain

$$
\varphi(z)=\varphi\left(f^{n_{k}} z\right)+\sum_{j=0}^{n_{k}-1} \gamma\left(f^{j}(z)\right)
$$

One can assume that $\varphi$ is bounded in the neighborhood $U:|\varphi(x)| \leq M, x \in \bar{U}$. Then (4.2) gives

$$
\sum_{i=1}^{k-1} \gamma\left(f^{n_{i}} z\right) \leq \sum_{j=0}^{n_{k}-1} \gamma\left(f^{j}(z)\right) \leq 2 M .
$$

Since $\sum_{i=1}^{k-1} \gamma\left(f^{n_{i}} z\right) \rightarrow \infty, k \rightarrow \infty$, we obtain a contradiction.

Corollary 4.5 Let $f: X \rightarrow$ Xbe a homeomorphism of a normal topological space. Then

$$
\Omega_{+}(f) \cup \Omega_{-}(f) \subset \mathcal{A}(f) .
$$

Indeed, let $(A, L)$ be some absorber for $H_{f}$. Set $\left(L_{0} \psi\right)(x)=-\psi\left(f^{n}(x)\right)$. Then $H_{f^{-1}} L_{0}=H_{f}$. Hence, the pair $\left(A, L_{0} L\right)$ is an absorber for $H_{f^{-1}}$.

\subsection{Applications to the Solvability Problem of Functional Equation}

Consider the functional equation

$$
(T \varphi)(x) \equiv P(x) \varphi(x)+Q(x) \varphi(f x)=\gamma(x), \quad(4.3)
$$

where $f: X \rightarrow X$ is a homeomorphism and

$$
\begin{aligned}
& P: X \rightarrow \operatorname{Hom}\left(\mathbb{C}^{n}, \mathbb{C}^{m}\right), \\
& Q: X \rightarrow \operatorname{Hom}\left(\mathbb{C}^{n}, \mathbb{C}^{m}\right), \\
& \gamma: X \rightarrow \mathbb{C}^{m}
\end{aligned}
$$

are given continuous mappings.

Assume that $\operatorname{rank} P(x)=m(x \in X)$. Then the pair $\left(A_{1}, L_{1}\right)$ where

$$
A_{1}=\overline{\bigcap_{\alpha} \bigcap_{n \geq q_{\alpha}} f^{n}\left(U_{\alpha}\right)}
$$

(see Example 2.6) and

$$
\left(L_{1} \psi\right)(x)=P^{*}(x)\left(P(x) P^{*}(x)\right)^{-1} \psi(x)
$$

is an absorber for $T$.

Analogously, assume that rank $B(x)=m(x \in X)$. Then the pair $\left(A_{2}, L_{2}\right)$, where

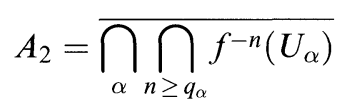

and

$$
\left(L_{2} \psi\right)(x)=Q^{*}(x)\left(Q(x) Q^{*}(x)\right)^{-1} \psi(x)
$$

is an absorber.

Let us call Eq. (4.2) nondegenerated if

$$
\operatorname{rank} P(x)=\operatorname{rank} Q(x)=m \quad(x \in X) .
$$

In this case solvability on intersection $A_{1} \cap A_{2}$ implies a global one, at least if $X$ is a normal topological space. In particular, if there are $A_{1}$ and $A_{2}$ with empty intersection, then (4.2) is solvable for any $\gamma$, i.e. $T$ is surjective. Moreover

THEOREM 4.6 Let $f: X \rightarrow X$ be a homeomorphism of a normal topological space. Then the following conditions are equivalent:

(a) the Abel equation

$$
\tau(x)-\tau(f(x))=1
$$

has a continuous solution $\tau \in C(X)$; 
(b) any nondegenerated equation (4.3) has a continuous solution $\varphi: X \rightarrow \mathbb{C}^{n}$;

(c) there exists an open countable covering $X=$ $\cup_{k=1}^{\infty} U_{k}, U_{k+1} \supset U_{k}$ such that

$$
f^{n}\left(\bar{U}_{k}\right) \cap \bar{U}_{k}=\emptyset, \quad n \geq n_{k} .
$$

If $X$ is a locally compact space countable at infinity, the condition (c) is equivalent to

(c') for any compact subset $K \subset X$ there is a number $n_{0}=n_{0}(K)$ such that

$$
f^{n}(K) \cap K=\emptyset, \quad n \geq n_{0} .
$$

The same result is true in classes of smooth vector functions on a smooth manifold $X$.

Theorem 4.6 was proved in [5]. For another application of our general approach to functional equations see $[1,2,3]$.

\section{References}

[1] G. Belitskii and V. Tkachenko, "On solvability of difference equations in smooth and real analytic vector functions of several variables". Integral Equations and Operator Theory, 18 (1994) 123-126.

[2] G. Belitskii and V. Nicolaevsky, "Linear functional equations of the line". Integral Equations and Operator Theory, 21 (1995) 212-223.

[3] G. Belitskii, "Smooth global solvability of first order differential equations." Diff. Eq. and Dyn. Syst., 3(2) (1995) 165-174.

[4] G. Belitskii and V. Nicolaevsky, "Extension theorem for linear functional-differential on the line", Functional Differential Equation, 3(1-2) (1995) 5-17.

[5] G. Belitskii and Yu. Lyubich, "The Abel equation and total solvability of functional equations" (preprint).

[6] R. Godement, Topologie Algebrique et Theorie des Faisceaux. Paris: Hermann, 1958, 283p.

[7] Yu. Lyubich, Linear Functional Analysis, Enc. of Math. Sci., Vol. 19, Berlin: Springer Verlag, 1992, 210 pp. 


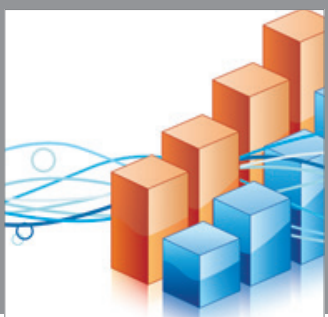

Advances in

Operations Research

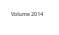

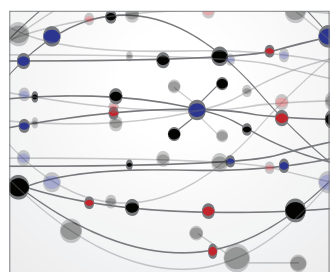

\section{The Scientific} World Journal
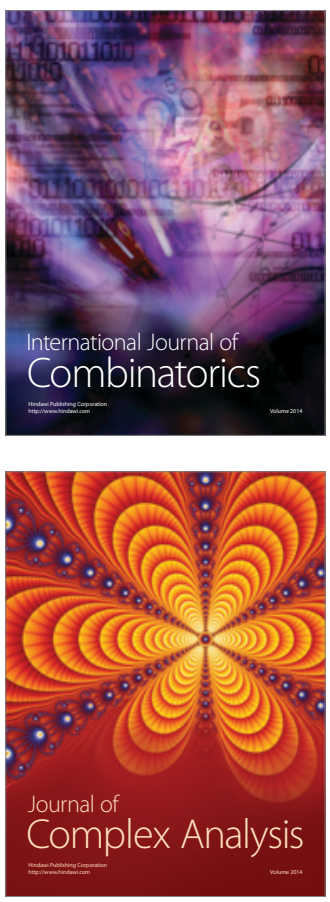

International Journal of

Mathematics and

Mathematical

Sciences
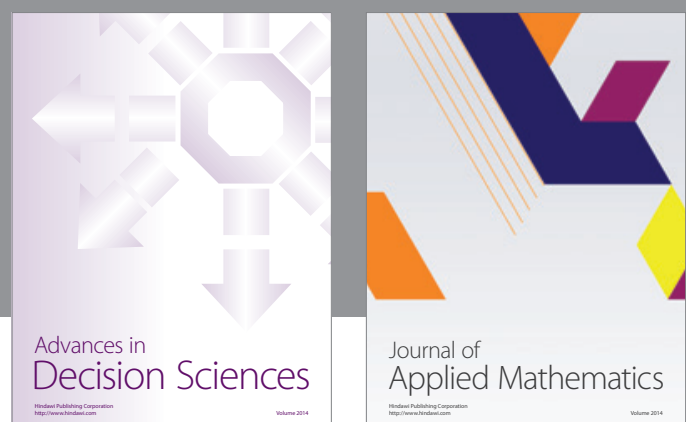

Journal of

Applied Mathematics
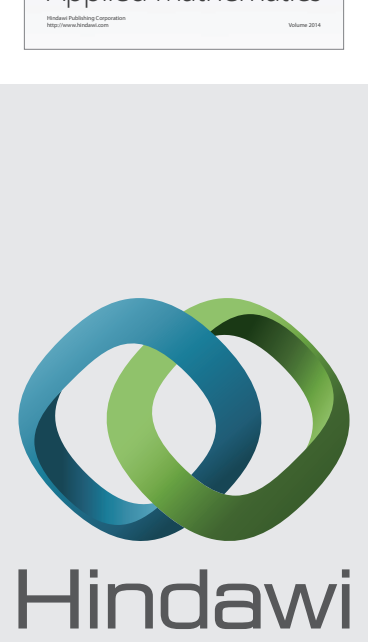

Submit your manuscripts at http://www.hindawi.com
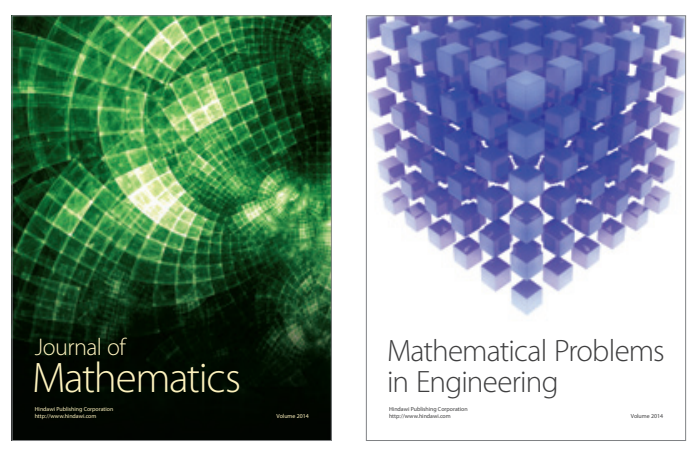

Mathematical Problems in Engineering
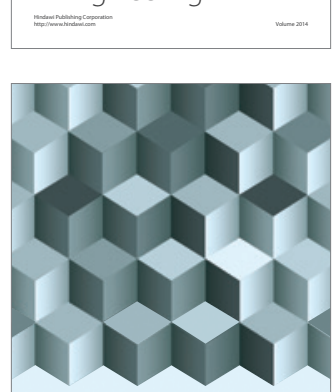

Journal of

Function Spaces
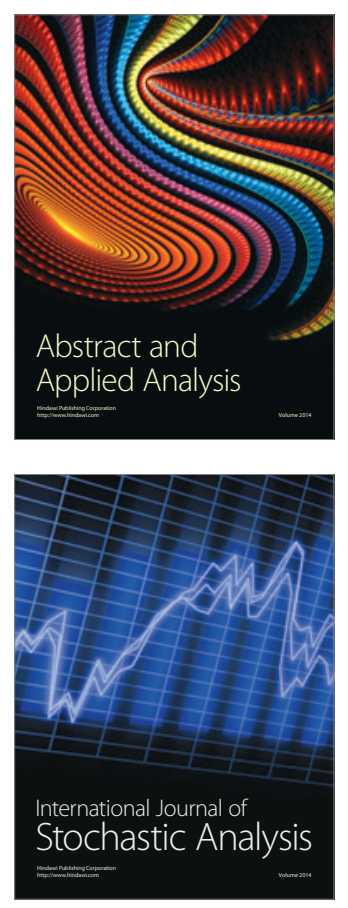

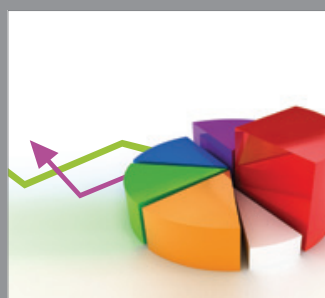

ournal of

Probability and Statistics

Promensencen
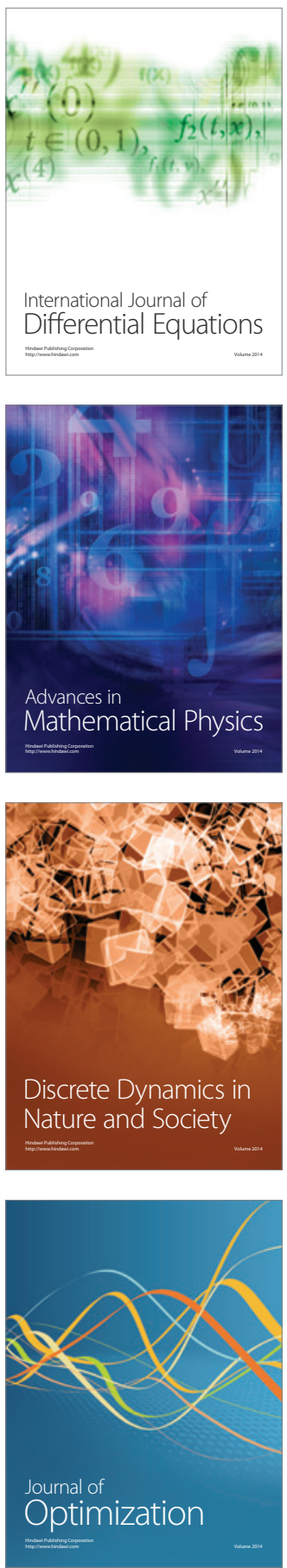